\title{
Analysis on Multi-direction Forging Defects of Railway Clamp Based on Certain Kind of Preformed Billet
}

\author{
Liying Liu ${ }^{1, a}$, Hongxia Yuan ${ }^{2, b}$ and Changbin Shao ${ }^{3, c}$ \\ 11,2,3Liying Liu, Beijing Research Institute of Mechanical \& Electrical Technology, China \\ alydially@sina.com, b13701356978@163.com, ${ }^{\mathrm{b}}$ 13466662616@139.com
}

Keywords: Railway clamp, multi-direction forging, numerical simulation, metal flow rule, forging defects

Abstract. The multi-direction forging process of railway clamp based on certain kind of preformed billet was designed. The railway clamp sample was acquired by industrial experiment. The causes of burr and other defects have been analyzed in multi-direction forging of railway clamp. The multi-direction forging process of railway clamp is simulated with DEFORM-3D software.Finite element analysis is developed to research the metal flow rule in forming process combined with the point tracing function in Deform. The result indicates that rough burr of railway clamp was mainly caused by the unreasonable design of experiment die structure. Forming load exceeds the capacity of the equipment, so the die is clamped badly. The critical reduction causing folding is $14 \mathrm{~mm} .9 \mathrm{~mm}$ reduction will not result in folding. The solution was proposed after the analysis of forging defects of railway clamp preformed billet and well-formed samples were got, which verified the rationality of preformed billet shape and feasibility of forming process.

\section{Introduction}

With the updating of railway operating system, the train speed is getting higher and higher. Raising speed puts forward higher requirements on stability and safety of railway track. Railway clamp is a high elastic railway fastener as shown in Fig.1[1], widely used in fixing high-speed railway tracks and sleeper. Railway clamp has large difference of cross section and thin baseplate. It is hard to reach the shape of this product by traditional forging method. At present, it is casted generally. In the other hand, it has many defects, such as segregation, shrinkage, uncertainty of properties, which may cause potential safety hazard so that the clamp could not satisfy the requirements for speeding up. Producing the high quality railway clamp is of great significance to develop the transportation in China. The multi-direction forging process of railway clamp is proposed combined with the unique advantage of multi-direction forging in forming complex and special-shaped products[2].

The sample of railway clamp is gained by industry experimentation through multi-direction forging. It has many defects affected by the unsuitable shape and size of preformed billet, and unsuitable structure of die. This paper researched the metal flow rule in multi-direction forging of railway clamp, the causes of forming defects was analyzed and the solution was put forward [3].

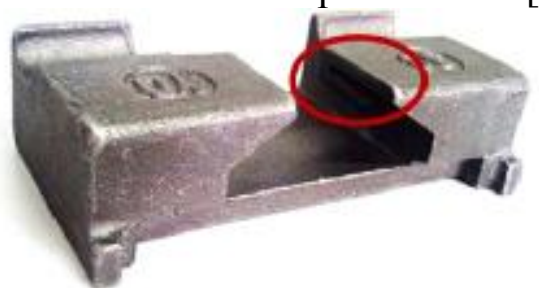

Fig.1 Railway clamp

\section{The multi-direction forging process of railway clamp and die structure}

Railway clamp can not demould well by single parting surface forging on account of the complexity of the product structure. So the multi-direction forging with more than one parting surface is presented according to the shape and structure of railway clamp, which is shown in Fig.2. Because large difference of cross section and huge metal deformation. 


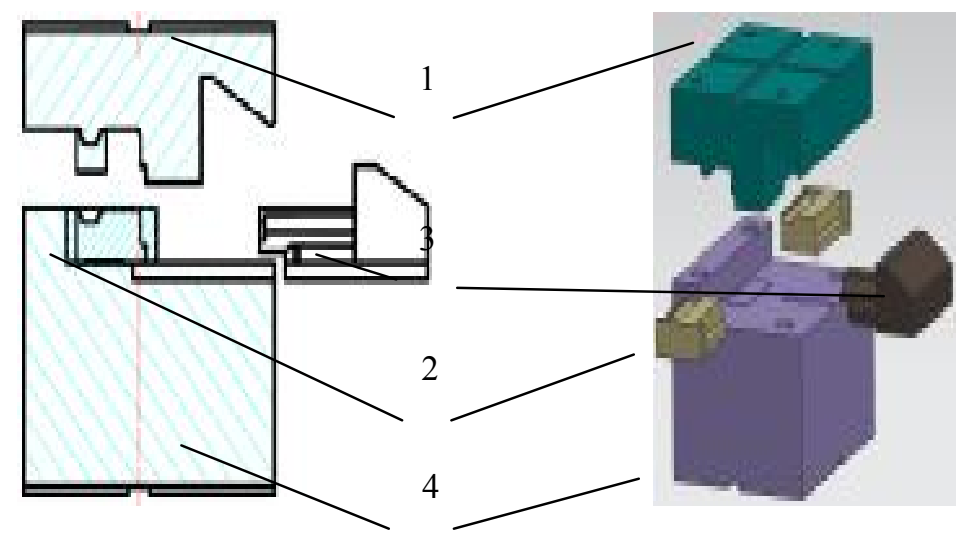

Fig. 2 Multi-dimensional forging die(a) and its physical model(b)

Industrial experiment on multi-direction forging process of railway clamp The preparation of experiment

Industrial experiment is carried on to test multi-direction forging process based on the structure of preformed billet. The preformed billet is machined with 45 steel square stock. The three-dimensional hydraulic press is used as testing equipment shown in Fig.3, which has $4000 \mathrm{KN}$ tonnage in both main cylinder and side cylinder. The preformed billet is heated by chamber electric furnace to $1150-1200{ }^{\circ} \mathrm{C}$. 1:10Water-based graphite is used as die lubricant through high pressure spray gun to lubricate the die. The die is preheated by blank roasting to about $100{ }^{\circ} \mathrm{C}$.

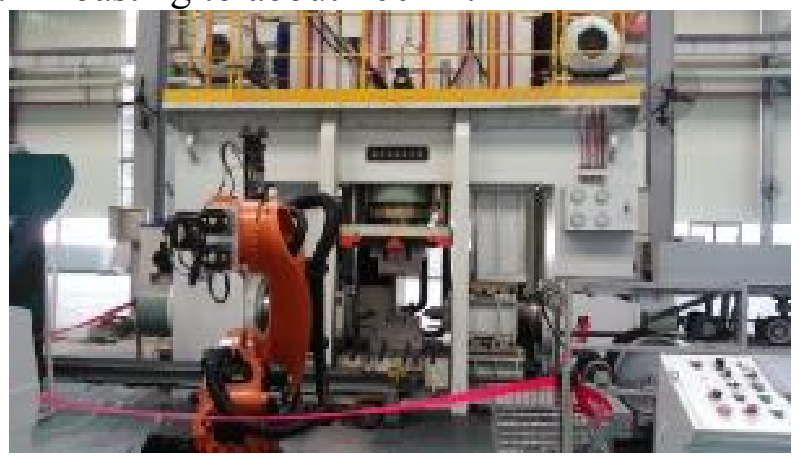

Fig.3 The three-dimensional hydraulic press

\section{Experimental result}

The railway clamp sample is acquired by industrial experiment in Fig.4. The picture indicates that defects of sample mainly includes serious burr in parting surface.

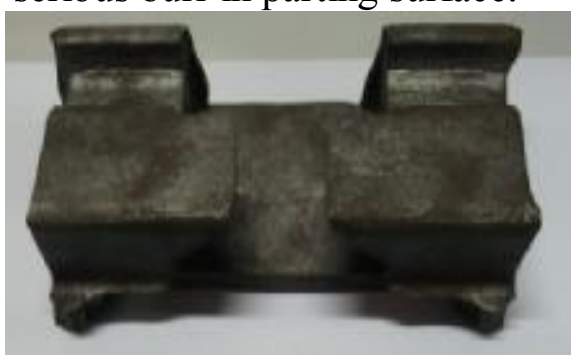

Fig.4 Sample of railway clamp

The burr in parting surface is shown in Fig.5. The Law of Minimum Resistance points out that metal flows along the minimum resistance dimension in plastic deformation process[4]. The burr usually emerged in the final stage of forging filling when the mental interstitial force is less than the mental flow resistance to other direction. The small burr can be removed by shot blasting generally. The thick burr in sample may be caused by much dislocation of die for insufficient tonnage of forming equipment. 


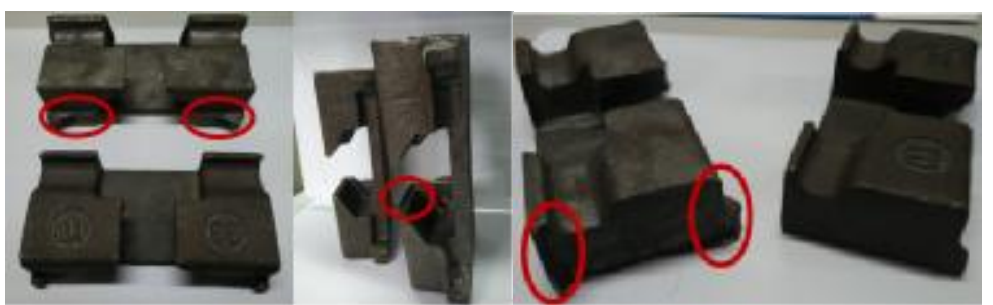

Fig. 5 Comparison diagram of sample forming defects with casting railway clamp

\section{The reason analysis of forming defects}

\section{The reason analysis of burr}

Through the above analysis, the basic reason of burr is dislocation of die for insufficient tonnage of forming equipment. Multi-dimensional forging force is influenced by projection area of workpiece, size of billet, friction coefficient, temperature, feed speed of die and other factors [5]. The finish-forging is simulated adopted the die in Fig.6a before test. The maximum clamp force is $3470 \mathrm{KN}$ in forging process, less than the maximum nominal pressure of equipment, which differs from the experimental result. The difference between simulation condition and actual working conditions includes friction coefficient and die structure through the analysis. 1:10water-based graphite lubricant is used in the experiment with friction coefficient in 0.04-0.20. Coulomb friction is often used in simulation, with friction coefficient in 0.18 . However direction of die clamping force is perpendicular to the friction, the dramatic increase of clamping force is not caused by the friction coefficient.

The structure of die used in experiment is shown in Fig6b. It is chosen because die should be clamping in four directions in finish-forging process. However it can only clamp manually unable to fix the position. Oblique mating surface structure is designed as Fig. $6 \mathrm{~b}$ to fix the side core die and avoid the dislocation. Oblique mating surface structure can fix the core die and increase the clamping force of upper die by receiving the force of side core die. The numerical simulation is carried out by Deform on die structure. And the curve about clamping force of upper die changing with time in forming process is shown in Fig.7. The curve A indicates the changing of clamping force of upper die in Fig.6a. The curve B shows the changing of clamping force of upper die in Fig.6b. The picture shows that the variation tendency of two curves is almost the same. The contribution oblique mating surface structure makes to increase the clamping force of upper die worth $3776.82 \mathrm{KN}$ by calculating the average of difference value at the same time. The simulation results demonstrate oblique mating surface structure adds requirements to the tonnage of equipment greatly. To decrease the forming load, the structure to locate the side core die is added in lower die base on the die structure in Fig.6a, which limits the forming tonnage into the test equipment capacity range successfully under simulation condition.

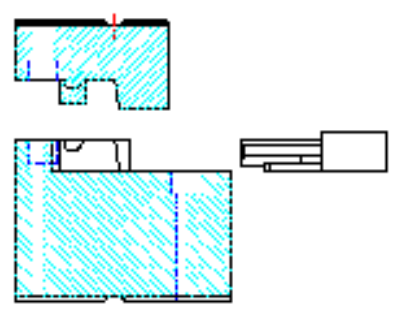

(a)

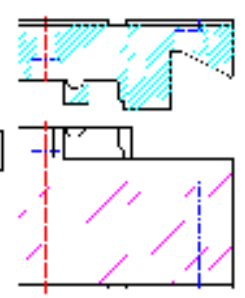

(b)

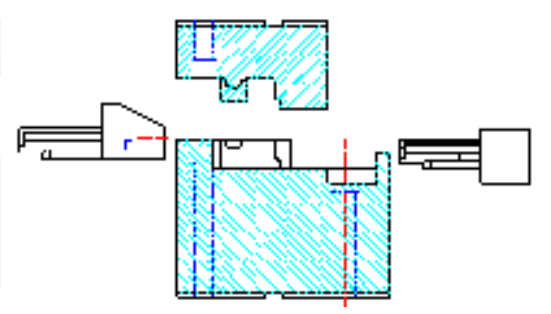

(c)

Fig. 6 Forming die

(a) Final forging die used for simulation before test (b)Final forging die used in experiment (c)Forming die after optimization 


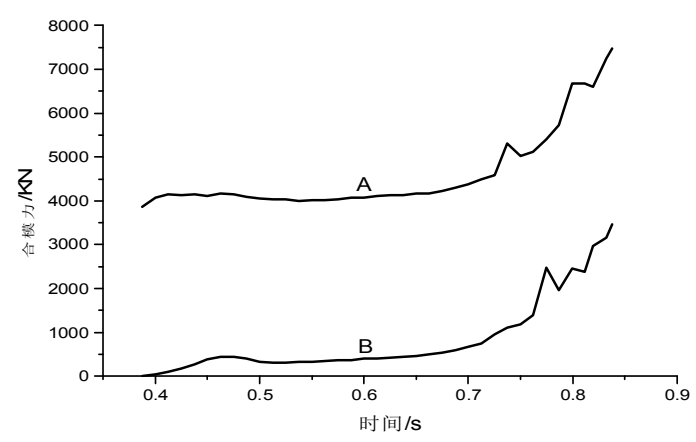

Fig. 7 Curves of clamping force for two different die

\section{Conclusion}

Through the analysis of numerical simulation on the multi-direction forging defects of railway clamp, it is concluded that the basic reason of burr in railway clamp sample is the dramatic increase of forming load caused by unreasonable design of die structure. The average of load increase reaches up to $3776.82 \mathrm{KN}$. Insufficient tonnage of equipment is solved by optimizing die structure and improving location way of die, in order to restrain burr. The solution is proposed with on the defects of railway clamp sample, after that well-formed sample is gained by simulation, to testify the feasibility of forming process, which has important guiding significance to present production.

\section{Reference:}

[1] Sheng W, Fu C F Types and Application of High-speed Railway Fastening System[J]. Metal Working, 2010, (7):31.35.

[2] Ren Y L, Niu L J, Cao F H, et al. Development of Multi-ram Forging Technology and Equipment[J]. Journal of Shanghai Jidian University, 2014,17(3):125-131.

[3] Xu L Q, Xiao M, Sun B S, et al. Study on Metal Flow Law of

High Neck Flange Forming by Closed Ring Rolling[J]. Light Industry

Machinery,2013,31(5):34-36,40.

[4] Xia J C, Wang X Y. Closed Die Forging[M] Beijing: China Machine Press, 2013.

[5] Xia J C, Wang X Y. Closed Die Forging[M] Beijing: China Machine Press, 2013. 